\title{
The use of 4D-CTA in the diagnostic work-up of brain arteriovenous malformations
}

\author{
Peter W. A. Willems • Patamintita Taeshineetanakul • \\ Barry Schenk • Patrick A. Brouwer • \\ Karel G. Terbrugge • Timo Krings
}

Received: 15 February 2011 / Accepted: 17 March 2011/Published online: 5 April 2011

(C) The Author(s) 2011. This article is published with open access at Springerlink.com

\begin{abstract}
Introduction We aimed to evaluate the use of time-resolved whole-head CT angiography (4D-CTA) in patients with an untreated arteriovenous malformation of the brain (bAVM), as demonstrated by catheter angiography (DSA).

Methods Seventeen patients with a DSA-proven bAVM were enrolled. These were subjected to 4D-CTA imaging using a 320 detector row CT scanner. Using a standardized scoring sheet, all studies were analyzed by a panel of three readers. This panel was blind to the DSA results at the time of reading the $4 \mathrm{D}-\mathrm{CTA}$.

Results 4D-CTA detected all bAVMs. With regard to the Spetzler-Martin grade, 4D-CTA disagreed with DSA in only one case, where deep venous drainage was missed. Further discrepancies between 4D-CTA and DSA analyses included underestimation of the nidus size in small lesions (four cases), misinterpretation of a feeding vessel (one case), misinterpretation of indirect feeding through pial collaterals (three cases) and oversight of mild arterial enlargement (two cases). 4D-CTA correctly distinguished low-flow from high-flow lesions and detected dural/ transosseous feeding (one case), venous narrowing (one case) and venous pouches (nine cases).

Conclusion In this series, 4D-CTA was able to detect all bAVMs. Although some angioarchitectural details were
\end{abstract}

P. W. A. Willems · P. Taeshineetanakul • K. G. Terbrugge

T. Krings $(\square)$

Division of Neuroradiology, Department of Medical Imaging,

Toronto Western Hospital, UHN,

399 Bathurst St., 3MCL-429,

Toronto, Ontario M5T 2S8, Canada

e-mail: timo.krings@uhn.on.ca

P. W. A. Willems $\cdot$ B. Schenk $\cdot$ P. A. Brouwer

Department of Radiology, Leiden University Medical Center, Leiden, The Netherlands missed or misinterpreted when compared to DSA, 4D-CTA evaluation was sufficiently accurate to diagnose the shunt and classify it. Moreover, 4D-CTA adds cross-sectional imaging and perfusion maps, helpful in treatment planning. 4D-CTA appears to be a valuable new adjunct in the noninvasive diagnostic work-up of bAVMs and their follow-up when managed conservatively.

Keywords Multi-detector row CT 320 slice CT AVM . Classification · Diagnosis

\section{Introduction}

Within the group of cranial vascular lesions, brain arteriovenous malformations (bAVMs) represent an abnormal communication between pial arteries supplying brain tissue and pial veins draining brain tissue [1]. Such abnormal communications can be as simple as to consist of a single fistulous connection, generally denoted a pial arteriovenous fistula, or consist of an extensive network of abnormal intervening vessels; a so-called nidus. The latter is usually of a compact or glomerular nature but may also be of a more diffuse or proliferative nature $[2,3]$.

The imaging characteristics of bAVMs will depend on various aspects of the lesion, such as the location, nature, and size of the nidus, whether or not recruited arterial feeders have increased in size to accommodate for the fistulous flow, whether or not the resulting fistulous flow has led to venous ectasias, whether the shunt interferes with blood supply to the brain by means of arterial steal or venous congestion, and whether prior bleeding has occurred. Many of these characteristics can be noted on planar imaging, such as computed tomography (CT) or magnetic resonance imaging (MRI), especially in larger 
lesions. However, the gold standard to demonstrate an arteriovenous shunt, which is necessary to demonstrate small lesions and to distinguish mimicking lesions like proliferative angiopathy $[3,4]$, is catheter-based digital subtraction angiography (DSA). DSA further helps to identify the involved feeding arteries and draining veins and to determine possible endovascular treatment strategies $[2,5]$. In addition, DSA can provide those angioarchitectural features that are important for treatment decision making.

Unfortunately, DSA is relatively expensive and timeconsuming and carries a rather high incidence of silent embolic events [6] and a small risk of transient or permanent neurologic deterioration [7-9]. Although the complication rate is most likely especially low in the relatively young, and otherwise healthy, AVM patient population, a noninvasive alternative angiographic method is of interest. Moreover, patients with a bAVM may undergo multiple angiographic evaluations over time. In recent years, MRI angiography (MRA) and CT angiography (CTA) have shown sufficient spatial resolution to answer many clinical neurovascular questions [10-13]. However, these vessel-cast techniques lacked the temporal resolution necessary to demonstrate an arteriovenous shunt (i.e., early venous filling) or to visualize feeding and draining patterns (e.g., high flow versus low flow shunts, presence or absence of venous rerouting).

More recently, time-resolved techniques have emerged for both MRA [14] and CTA [15, 16]. Aside from generating cross-sectional images, these datasets enable visualization of blood flow dynamics in cranial vessels with the first pass of an intravenous (IV) contrast bolus. Hence, it is conceivable that they may replace DSA in cases in which time-resolved imaging is required. We aimed to evaluate the advantages and disadvantages of whole head time-resolved CTA (4D-CTA) in the clinical setting of a consecutive series of patients with an untreated bAVM, for whom DSA imaging was already available.

\section{Materials and methods}

Patient selection and data collection

Approval for this study was obtained from our institutional research ethics board in November 2008, and patients were admitted to the study between November 2008 and March 2010. Patients were included if DSA demonstrated an untreated bAVM. Exclusion criteria were patient age of below 18 years, current treatment for diabetes mellitus, known allergy for iodinated contrast agents, and renal failure (indicated by a baseline serum creatinine above $133 \mu \mathrm{mol} / \mathrm{l})$.
After obtaining informed consent, 4D-CTA imaging was performed. All DSA and 4D-CTA studies were anonymized and a panel of three readers (authors PW, PT, and TK) performed a consensus reading. For each patient, the panel was blinded for the DSA results at the time of reading the 4D-CTA. Subsequently, a standardized scoring sheet was filled out for each diagnostic study (see below). Furthermore, we documented demographic and clinical data for each patient.

\section{DSA examination}

Diagnostic intra-arterial DSA was performed with standard biplane fluoroscopy equipment (Infinix, Toshiba Medical Systems, Japan; or LCL-P, GE Healthcare, UK). Since patients were only included in the study after DSA had demonstrated a bAVM, the angiographic protocol was not influenced by the study. It generally consisted of bilateral injections of the internal carotid arteries (ICA), external carotid arteries (ECA) and at least one vertebral artery (VA). During each injection anterior-posterior (AP) and lateral projections were obtained at $3 \mathrm{fps}$.

\section{D-CTA examination}

All 4D-CTA examinations were performed using an Aquilion ONE multidetector CT scanner (Toshiba Medical Systems, Japan), equipped with $320 \times 0.5 \mathrm{~mm}$ detector rows covering $16 \mathrm{~cm}$ of volume per rotation. Imaging was performed in a manner previously described $[16,17]$. In short, intravenous infusion of $60 \mathrm{ml}$ of nonionic contrast medium, followed by $20 \mathrm{ml}$ of saline, was followed by a dynamic acquisition sequence with a gantry rotation speed of 1 rotation per second. The dynamic acquisition sequence consisted of one mask volume $(80 \mathrm{kVp}, 300 \mathrm{mAs})$ and 22 dynamic volumes $(80 \mathrm{kVp}, 120 \mathrm{mAs})$. After subtracting the mask volume from the dynamic volumes, a total of 7,040 $(22 \times 320)$ images were stored in DICOM files. Using these files, the standard scanner software generated time-resolved

Table 1 Distribution of presenting symptoms of the patients enrolled in this study

\begin{tabular}{lr}
\hline Presentation & $n$ \\
\hline Hemorrhage & 4 \\
Epilepsy & 5 \\
Neurologic deficit & 2 \\
Headache & 1 \\
Incidental & 3 \\
Unknown & 2 \\
Total & 17 \\
\hline
\end{tabular}


Table 2 Items scored by the reading panel for each diagnostic study and results of both 4D-CTA and DSA

\begin{tabular}{|c|c|c|c|}
\hline Item & Options & 4D-CTA & DSA \\
\hline Shunt detected (early venous filling) & Yes/no & 17 & 17 \\
\hline bAVM diagnosed & Yes/no & 17 & 17 \\
\hline \multicolumn{4}{|l|}{ Spetzler-Martin grade ${ }^{\mathrm{b}}$} \\
\hline \multirow[t]{3}{*}{ Size } & (1) $<3 \mathrm{~cm}$ & 12 & 12 \\
\hline & (2) $3-6 \mathrm{~cm}$ & 5 & 5 \\
\hline & (3) $>6 \mathrm{~cm}$ & 0 & 0 \\
\hline \multirow[t]{2}{*}{ Eloquence } & (0) Non-eloquent location & 5 & 5 \\
\hline & (1) Eloquent location & 12 & 12 \\
\hline \multirow[t]{2}{*}{ Drainage } & (0) Superficial drainage only & $10^{\mathrm{c}}$ & 9 \\
\hline & (1) Deep drainage present & $7^{\mathrm{c}}$ & 8 \\
\hline \multirow[t]{3}{*}{ Arterial feeding territories } & Anterior circulation (AC) & 15 & 15 \\
\hline & Posterior circulation (PC) & $5^{\mathrm{d}}$ & 6 \\
\hline & (or both) & $(3)^{\mathrm{d}}$ & (4) \\
\hline \multirow[t]{12}{*}{ Arterial feeders } & $\mathrm{AC}$ & & \\
\hline & Deep branches from the anterior cerebral artery & 1 & 1 \\
\hline & Cortical branches from the anterior cerebral artery & $5^{\mathrm{e}}$ & 4 \\
\hline & Deep branches from the middle cerebral artery & $5^{\mathrm{d}}$ & 4 \\
\hline & Cortical branches from the middle cerebral artery & $10^{\mathrm{f}}$ & 11 \\
\hline & $\begin{array}{l}\text { Branches from the internal carotid artery, anterior choroidal artery } \\
\text { or ophthalmic system } \\
\text { PC }\end{array}$ & 0 & 0 \\
\hline & Branches from the vertebral artery or PICA & 0 & 0 \\
\hline & Branches from the basilar artery or AICA & 0 & 0 \\
\hline & Branches from the superior cerebellar artery & 1 & 1 \\
\hline & Deep branches from the posterior cerebral artery & $1^{\mathrm{d}}$ & 2 \\
\hline & Cortical branches from the posterior cerebral artery & 3 & 3 \\
\hline & (or any combination of these) & - & - \\
\hline Dural artery supply & Yes/no & 1 & 1 \\
\hline Watershed transfer & Yes/no & $3^{\mathrm{e}, \mathrm{g}}$ & 6 \\
\hline Arterial enlargement & Yes/no & $10^{\mathrm{h}}$ & 12 \\
\hline Flow related aneurysm(s) & Yes/no & 0 & 0 \\
\hline Nidus type & Micro $(<1 \mathrm{~cm})$ or fistula/macro $(>1 \mathrm{~cm})$ & $5 / 12^{\mathrm{i}}$ & $1 / 16$ \\
\hline bAVM flow & Low flow/high flow & $6 / 11$ & $6 / 11$ \\
\hline Intranidal stasis of contrast & Yes/no & 0 & 0 \\
\hline Venous outflow obstruction & Yes/no & 0 & 0 \\
\hline Venous pouch(es) & Yes/no & 9 & 9 \\
\hline
\end{tabular}

PICA posterior inferior cerebellar artery, AICA anterior inferior cerebellar artery

a In "yes/no" items, $n$ represents the number of patients who scored "yes"

${ }^{\mathrm{b}}$ Since we were only interested in the diagnostic performance of each study, we excluded the Spetzler-Martin category "inoperable" (6)

${ }^{\mathrm{c}} 4 \mathrm{D}$-CTA missed deep venous drainage in one patient

${ }^{\mathrm{d}}$ In one case, the posterolateral choroidal artery feeding the bAVM was mistakenly interpreted as a lenticulostriate perforator in the 4D-CTA

${ }^{\mathrm{e}}$ Indirect feeding through leptomeningeal collaterals (watershed transfer) was erroneously interpreted as direct feeding in one case in 4D-CTA

${ }^{\mathrm{f}}$ Cortical branches of the anterior and middle cerebral artery feeding the bAVM were interpreted by 4D-CTA to be from the anterior cerebral artery only, in one case

${ }^{\mathrm{g}}$ Watershed transfer with hypoxemia induced nonsprouting angiogenesis was missed by 4D-CTA in three cases: these vessels were too small, interpreted as direct feeders (see footnote "e") or interpreted as part of the nidus (one case each)

${ }^{\mathrm{h}}$ Arterial enlargement of feeding vessel(s) was too mild too be noticed by 4D-CTA in two cases

${ }^{\mathrm{i}} 4 \mathrm{D}-\mathrm{CTA}$ underestimated nidus size in 4 cases, interpreting them as micro $(<1 \mathrm{~cm})$ whereas DSA demonstrated them to be macro $(>1 \mathrm{~cm})$ 
(arterial to venous) maximum intensity projections (MIPs) at different viewing angles.

Angiographical evaluation

Both the 4D-CTA examinations and the DSA examinations were scored with the same scoring sheet. Items to be scored included presence or absence of a shunt, grading according to the Spetzler-Martin grade (size, eloquence of the location, presence of deep venous drainage) but also additional angioarchitectural features that are routinely reported upon in our institutions when reading out bAVM studies on DSA. These included for the arterial aspect of the angiographies: feeding arteries, presence of dural supply, arterial enlargement, flow-related aneurysms and presence of a transfer of the watershed (i.e., indirect supply to an arterial territory via leptomeningeal collaterals). Concerning the nidus, we evaluated the nidus type (micro bAVM or fistula versus macro bAVM) and the flow volume; concerning the venous aspect of the angiographies stagnation of contrast material, venous outflow obstruction and the presence of venous pouches were sought after.

\section{Results}

Between November 2008 and March 2010, 17 consecutive patients with an untreated bAVM were included, 6 male and 11 female. Their mean age $( \pm \mathrm{SD})$ was $38( \pm 17)$ years. Table 1 shows the distribution of presenting symptoms and Table 2 shows the characteristics of the bAVMs within our patient group, according to the items in the standardized scoring sheet, with the results of 4D-CTA and DSA analysis. Of the four patients who presented with hemorrhage, only one was imaged in the acute phase. There was only minor structural compromise by the hematoma in this case. The other three were imaged 4 months, 11 months, and 5 years after hemorrhage.

All 17 bAVMs were detected with 4D-CTA. With regard to the Spetzler-Martin grade, there was disagreement in one case, where deep drainage was not detected with 4D-CTA. Interestingly, four of the remaining seven cases with deep venous drainage showed this deep drainage to be indirect from rerouting of cortical veins draining the bAVM, all of which were correctly identified with 4D-CTA (Fig. 1).

Though there was agreement in all cases in the size classification used in the Spetzler-Martin grade, the subclassification of small bAVMs into micro bAVMs $(<1 \mathrm{~cm}$, including pial fistulas) versus macro bAVMs $(>1 \mathrm{~cm})$ was not agreed upon in all cases (Fig. 2). Five lesions were denoted micro based on the 4D-CTA, while DSA identified only a single fistulous micro bAVM whereas the other four lesions were noted to be small bAVMs with a nidus size larger than $1 \mathrm{~cm}$.
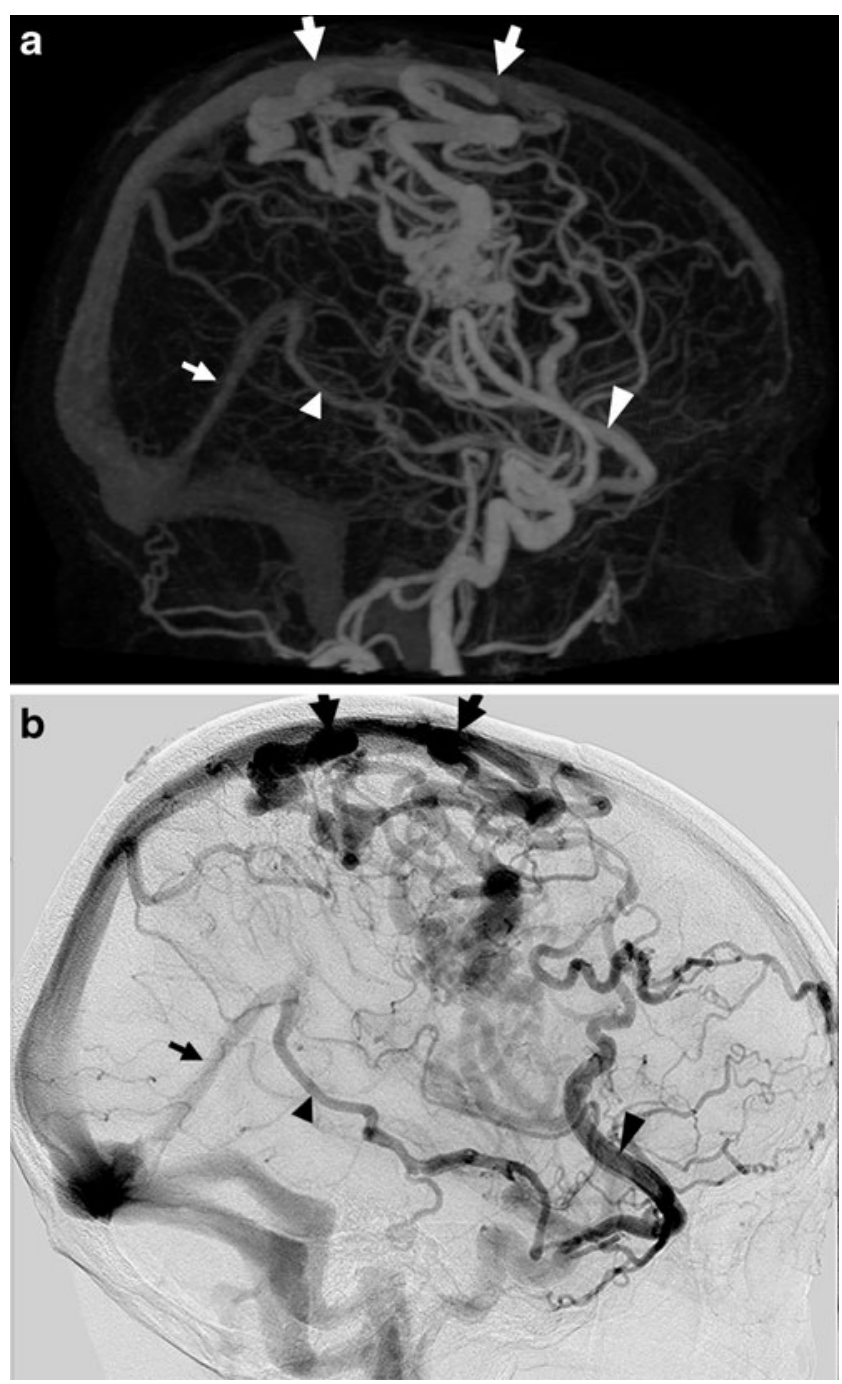

Fig. 1 Example of a patient with a Spetzler-Martin grade 3 bAVM with indirect deep venous drainage, recognized in the lateral projection of the 4D-CTA MIP (a) and the DSA (b). Early drainage to the superior sagittal sinus (large arrows) precedes indirect drainage to the straight sinus (small arrow) through the Sylvian vein (large arrowhead) and the basal vein of Rosenthal (small arrowhead). Though this can be recognized in these images through differences in opacification, the analysis is simplified when studying the entire timeresolved series

With regard to arterial feeding territories (anterior and/or posterior circulation), there was disagreement in one patient. In this case, the posterolateral choroidal artery feeding the bAVM was mistakenly interpreted as a lenticulostriate perforator in the 4D-CTA (Fig. 3). Looking at the feeding branches within the anterior and posterior circulation, discrepancies were found in two additional patients. One of these demonstrated indirect feeding from the anterior cerebral artery through pial collaterals to the middle cerebral artery territory. This was interpreted as direct feeding in the 4D-CTA. The other showed a lesion fed by cortical branches of the anterior and middle cerebral 

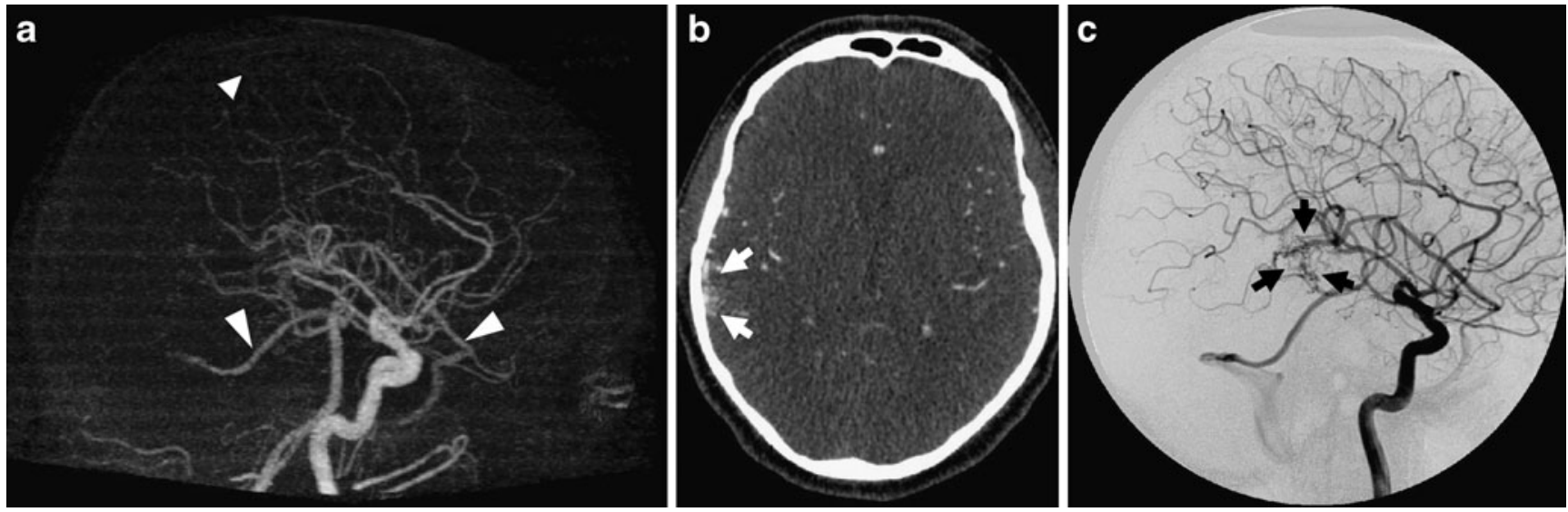

Fig. 2 Example of a patient with a small Spetzler-Martin grade 1 bAVM, superficially in the right temporal area. Based on the 4D-CTA the nidus was scored as micro $(<1 \mathrm{~cm})$ whereas the DSA demonstrated a macro nidus $(>1 \mathrm{~cm})$. Lateral MIP of the arterial phase of the $4 \mathrm{D}$ CTA (a) demonstrates two obvious draining veins (large arrowheads) and one faint one (small arrowhead). The nidus, however, cannot clearly be discerned. An axial slice at the level of the presumed nidus

artery, while the 4D-CTA suggested feeding from cortical branches of the anterior cerebral artery only.

Secondary dural and transosseous feeding of the bAVM was only seen in one patient and was correctly detected in the 4D-CTA (Fig. 4). Arterial enlargements, indicating feeding vessels, were seen in 12 cases, but were too mild to be noticed in the 4D-CTA in two of these. Indirect feeding through enlarged leptomeningeal collaterals from one cortical territory to another, also referred to as watershed in the same phase (b) suggests the nidus to be larger than suggested by the MIP (arrows). Lateral projection of the late arterial phase of the DSA (c) demonstrates the nidus more clearly (arrows). The rather diffuse nature of the nidus, possibly due to disruption by the presenting hemorrhage 4 months earlier, was thought to be the cause of poor detection by $4 \mathrm{D}-\mathrm{CTA}$

transfer related to hypoxemia induced nonsprouting angiogenesis [2], could be seen in six cases, but were only detected in the 4D-CTA of three of these (Fig. 5). In the 4D-CTA of the other three, these indirect feeders were either too small to be noticed, interpreted as direct feeders or interpreted as part of the nidus (one case each).

4D-CTA and DSA agreed on flow type in all cases, judging six bAVMs to be "low flow" and 11 to be "high flow". Neither 4D-CTA nor DSA detected intranidal stasis
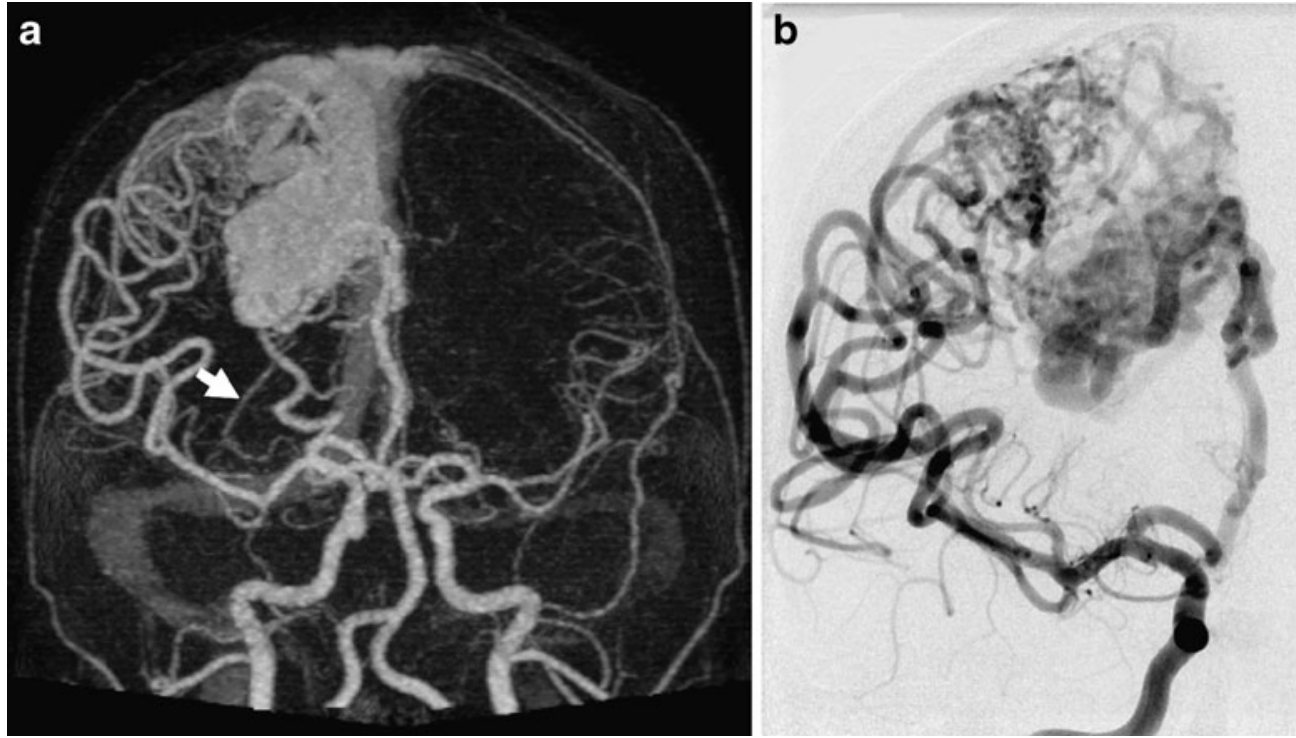

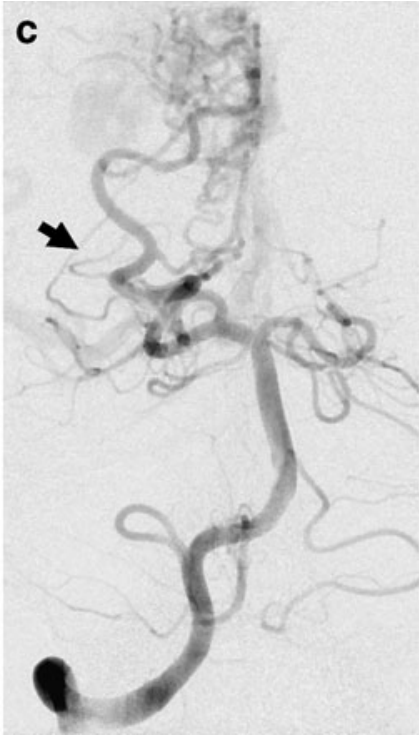

Fig. 3 Example of a patient with a Spetzler-Martin grade 3 bAVM in the right parietal lobe. A 4D-CTA MIP in the arterial phase (a) suggests an enlarged lenticulostriate perforator (arrow) feeding the deeper part of the nidus. The right ICA injection of the DSA in the same phase and projection (b) does not confirm this feeder. The right
VA injection of the DSA in the same phase and projection (c) shows the vessel to be the right posterolateral choroidal artery (arrow). The false interpretation of the 4D-CTA, even when oblique MIPs were used, is due to the non-selective opacification of all cranial arteries with this technique 

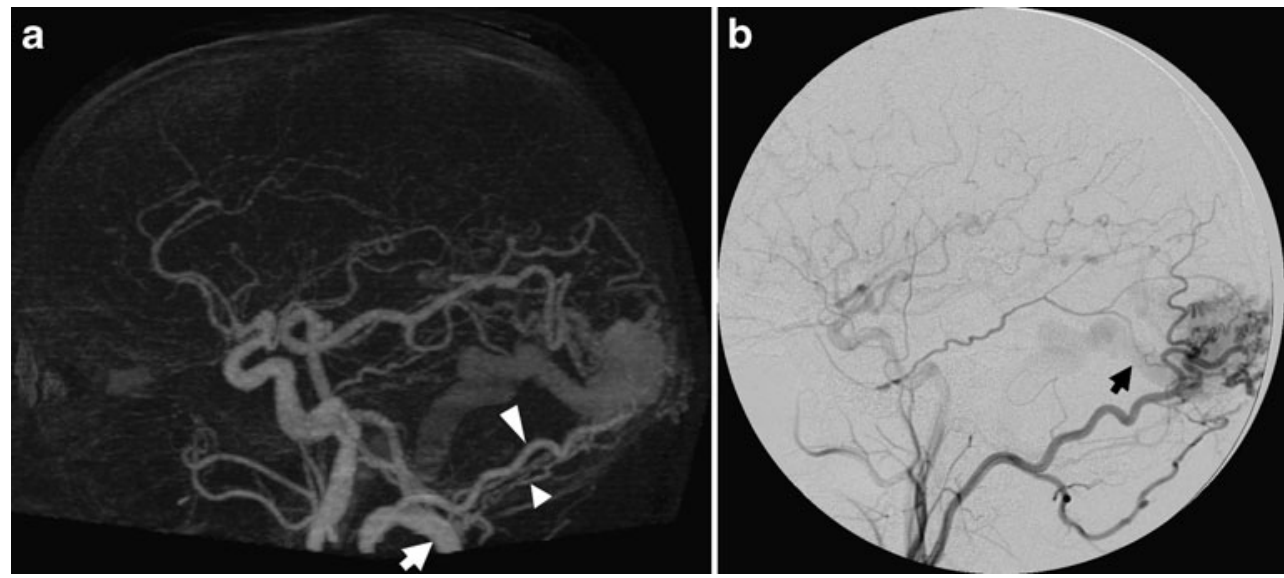

Fig. 4 Images of the only patient in our series with secondary dural and transosseous feeding of a bAVM. The lateral MIP of the 4D-CTA in early arterial phase (a) clearly shows an enlarged occipital artery (large arrowhead) running up to the lesion, as well as smaller posterior meningeal branches (small arrowhead) from the ipsilateral

of contrast material in any of the lesions. Both modalities showed significant venous stenosis in only one case (Fig. 6) and both agreed on venous pouches being present in nine patients. Venous obstruction, i.e., occlusion of a dural sinus or internal jugular vein, was not detected in any patient.

\section{Discussion}

We aimed to offer an evaluation of the performance of time-resolved CTA in the diagnostic work-up of untreated bAVMs and recognize its potential capabilities and pitfalls in the evaluation of angioarchitectural details deemed to be important for the possible treatment strategies.

To date, the major drawbacks of non-invasive timeresolved vascular imaging are the inferior spatial and temporal resolution compared to DSA. With time-resolved MRA (trMRA), there is a trade-off between spatial resolution and temporal resolution [18]. With 4D-CTA, temporal and spatial resolution are independent parameters; but there is a trade-off between temporal resolution, signal-to-noise ratio and radiation dose. For the 4D-CTA protocol used in this study, radiation dose was $5.2 \mathrm{mSv}$ or less [16]. With further refinements of imaging parameters or acceptance of dose increase, 4D-CTA results may improve further. Even with current protocols, however, a combination of $1 \mathrm{~s}$ image intervals and $0.5 \mathrm{~mm}$ isotropic voxel size renders 4D-CTA superior to current trMRA.

A comparison of radiation dose between 4D-CTA and DSA is not easy, since the radiation dose received during DSA is highly variable. It depends on the fluoroscopy time per vessel studied, the number of vessels studied, fluoroscopic parameters, type of fluoroscopic equipment, and so
VA (arrow). Differentiation of a purely dural AV shunt is possible, however, due to depiction of the enlarged posterior cerebral artery feeding an intraparenchymal nidus. The lateral projection of the ECA injection (b) proves the ECA contribution, by opacification of the bAVM and early opacification of the draining vein (arrow)

forth. We calculated the radiation dose of four typical cerebral DSAs, performed at our center. These varied between 7.89 and $9.12 \mathrm{mSv}$. The calculations were based on Monte Carlo simulations by using the dose-area product values and beam geometry information. Ninety-five percent of this dose was estimated to have been delivered to the cranium, with minor exposure of the thorax, abdomen, and pelvis.

The present comparison with DSA demonstrated that early venous filling was recognized by 4D-CTA in all cases, independent of nidus size. In addition, the correct distinction between the type of feeding artery (dural versus pial arteries) could be made. Although the present series did not include other shunting lesions (such as dural AV fistulae) or normal controls, the correct identification of these two features, suggests that 4D-CTA can correctly identify the type of shunting lesion in most instances. However, the drawbacks of 4D-CTA came into play when evaluating the angioarchitecture of each lesion in finer detail, as has also been shown in other types of arteriovenous shunting lesions [19]. Not only did such detailed evaluation suffer from impaired spatial resolution, temporal resolution and signal-to-noise ratio, but also from non-selectivity of the vessel opacification. This nonselectivity eliminates the ability to isolate certain vessels and leads to a "crowded" appearance of the vasculature. As a consequence, it is more difficult to determine the nature of certain vessels and their relationship to the lesion at hand.

In the present series, these characteristics of 4D-CTA led to misinterpretation of a feeding vessel (one case), misinterpretation of indirect feeding through pial collaterals (three cases), oversight of mild arterial enlargement (two cases) and oversight of deep venous drainage (one case). 

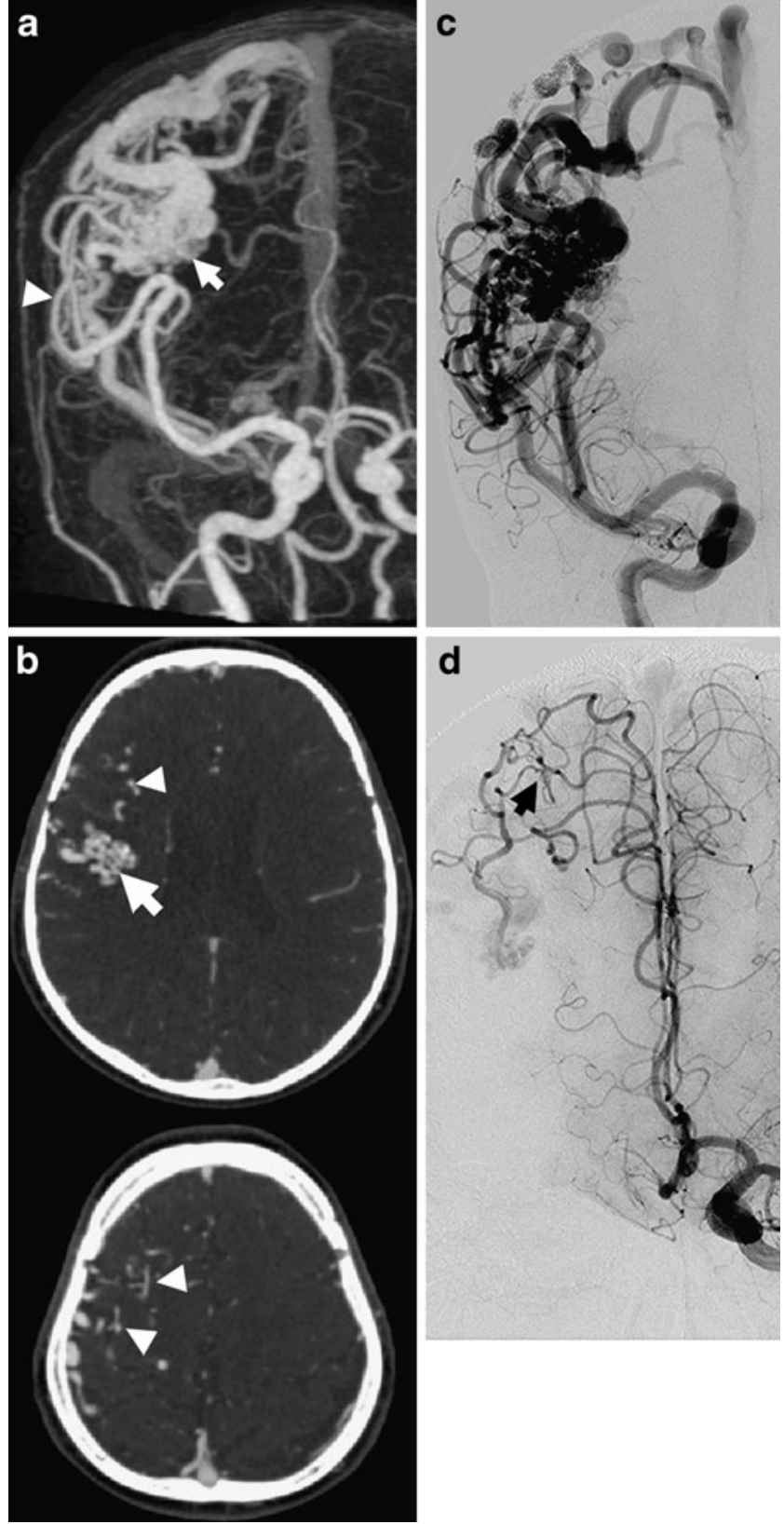

Fig. 5 Example of a patient with a Spetzler-Martin grade 3 bAVM (same case as Fig. 1) illustrating superiority of DSA over 4D-CTA to demonstrate indirect feeding of a bAVM, i.e., watershed transfer. The AP MIP of the 4D-CTA (a) depicts a right frontal bAVM (arrow), fed by branches from the middle cerebral artery (arrowhead). Axial images from the 4D-CTA in early venous phase (b) at the level of the nidus (upper image) and above the level of the nidus (lower image) allow identification of the compact nidus (arrow) and feeding vessels (arrowheads), but identification of indirect feeders remains challenging. AP DSA images after injection of the right ICA (c) and left ICA (d) clearly aid this discrimination by demonstrating recruitment of leptomeningeal anastomoses (arrow) which have developed to enhance pial collateral supply from the anterior to the middle cerebral artery

Furthermore, 4D-CTA interpreted four bAVMs to be smaller than the estimation based on DSA $(<1 \mathrm{~cm}$ rather than $>1 \mathrm{~cm}$ ). However, large inter-observer discrepancies in nidus delineation are known to occur with DSA as well [20].

We believe evaluation of the cross-sectional 4D-CTA (source) images is essential when reading 4D-CTA data, since these images may contain information which has been lost in subsequent 3D reconstructions. 4D-CTA crosssectional images may also be analyzed in a time-resolved fashion and may be helpful to recognize the nidus (Fig. 2) and distinguish it from surrounding feeders (Fig. 5). Moreover, they can be used to observe structural changes not directly visible in the MIPs or in DSA imaging, such as hydrocephalus or a hematoma, and may aid surgical planning. Other additional image sets that can be reconstructed from the same dynamic imaging sequence are perfusion maps [17], which may be used to assess the microcirculation of tissues surrounding the bAVM. This microcirculation may be impaired by vascular steal phenomena or venous hypertension, which may impact on planning and risk estimation of bAVM treatment [21, 22].

Although the capabilities of 4D-CTA in the assessment of bAVMs appear promising, we feel caution is appropriate when attempting to change present imaging paradigms. Firstly, no conclusion can be drawn with regard to flow related arterial aneurysms or intranidal (pseudo-) aneurysms, which may represent a target for endovascular treatment after intracranial hemorrhage [2,5], since neither were present in this series. Secondly, in our previous study regarding dural arteriovenous fistulas [19], a single lesion was missed due to its slow flow and the crowded appearance of the 4D-CTA. It is conceivable that such errors may also occur when analyzing bAVMs, especially when small lesions with a low shunt volume are present. This question can only be answered by an unselected patient cohort in a prospective study that includes patients in whom a DSA is deemed necessary to rule out a shunting lesion so as to include patients with different types of pathology. Until such a study is published, we suggest to use 4D-CTA only as either a screening tool in the work-up of suspected patients, followed by DSA whenever negative, or as a follow-up tool when a lesion has been demonstrated previously and is managed conservatively.

\section{Conclusion}

In this series of bAVM patients, 4D-CTA was able to detect all lesions. Although some angioarchitectural details were missed or misinterpreted when compared to DSA, 4D-CTA evaluation would have been sufficiently accurate to diagnose the shunt and classify it. We believe treatment planning may benefit from the accompanying availability of cross-sectional imaging in different planes 
Fig. 6 Images of the only patient in our series with venous stenosis. The AP MIP of the 4D-CTA (a) and the DSA image in AP projection after left ICA injection (b), both in late arterial phase, demonstrate the single arterial feeding branches (arrows), the level of the pial fistula (asterisk) and multiple draining veins (small arrowheads) draining into the superior sagittal sinus. The largest draining vein narrows significantly before reaching the sinus (large arrowhead)
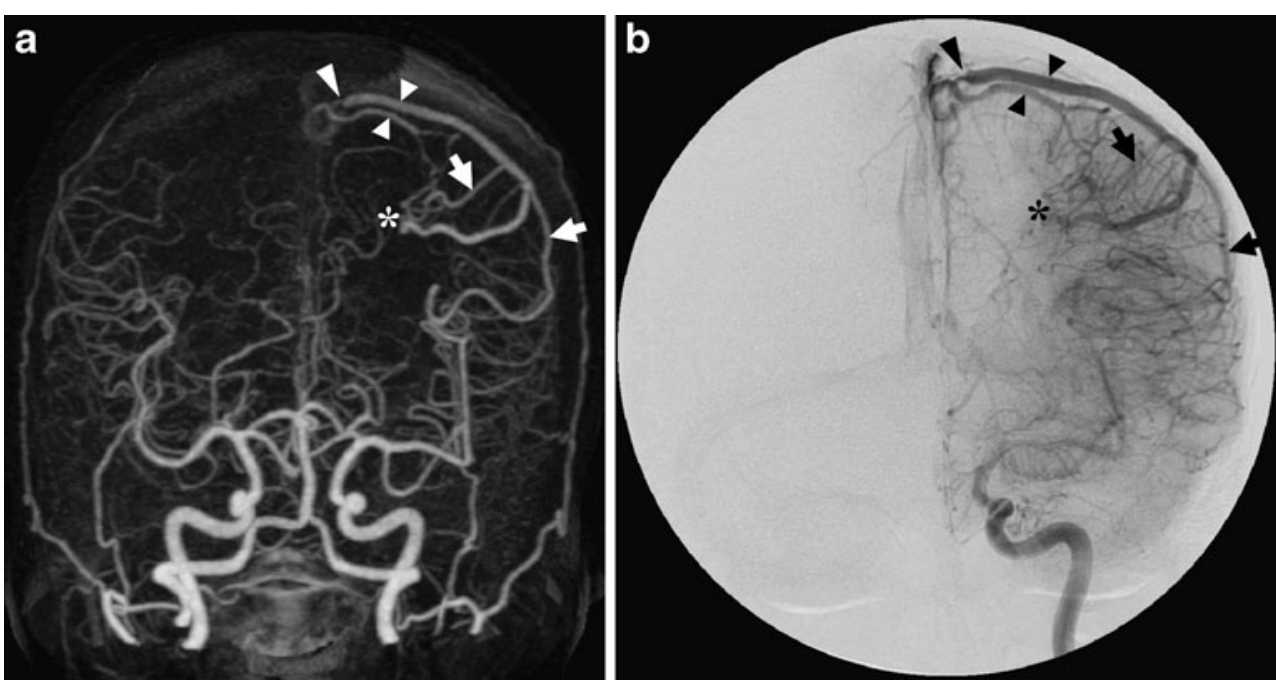

and perfusion maps, which can be calculated from the presented data set. Although these results await validation in a prospective unselected patient cohort, 4D-CTA seems to be a valuable new adjunct in the non-invasive diagnostic work-up and follow-up of bAVM patients.

Conflict of interest We declare that we have no conflict of interest.

Open Access This article is distributed under the terms of the Creative Commons Attribution Noncommercial License which permits any noncommercial use, distribution, and reproduction in any medium, provided the original author(s) and source are credited.

\section{References}

1. Friedlander RM (2007) Clinical practice. Arteriovenous malformations of the brain. N Engl J Med 356:2704-2712

2. Berenstein A, Lasjaunias P, terBrugge KG (2004) Surgical neuroangiography. 2.1. Clinical and endovascular treatment aspects in adults. Springer, Berlin

3. Geibprasert S, Pongpech S, Jiarakongmun P, Shroff MM, Armstrong DC, Krings T (2010) Radiologic assessment of brain arteriovenous malformations: what clinicians need to know. Radiographics 30:483-501

4. Lasjaunias PL, Landrieu P, Rodesch G, Alvarez H, Ozanne A, Holmin S, Zhao WY, Geibprasert S, Ducreux D, Krings T (2008) Cerebral proliferative angiopathy: clinical and angiographic description of an entity different from cerebral AVMs. Stroke 39:878-885

5. Krings T, Hans FJ, Geibprasert S, TerBrugge K (2010) Partial "targeted" embolisation of brain arteriovenous malformations. Eur Radiol 20:2723-2731

6. Bendszus M, Koltzenburg M, Burger R, Warmuth-Metz M, Hofmann E, Solymosi L (1999) Silent embolism in diagnostic cerebral angiography and neurointerventional procedures: a prospective study. Lancet 354:1594-1597

7. Cloft HJ, Joseph GJ, Dion JE (1999) Risk of cerebral angiography in patients with subarachnoid hemorrhage, cere- bral aneurysm, and arteriovenous malformation: a metaanalysis. Stroke 30:317-320

8. Kaufmann TJ, Huston J 3rd, Mandrekar JN, Schleck CD, Thielen KR, Kallmes DF (2007) Complications of diagnostic cerebral angiography: evaluation of 19,826 consecutive patients. Radiology 243:812-819

9. Willinsky RA, Taylor SM, TerBrugge K, Farb RI, Tomlinson G, Montanera W (2003) Neurologic complications of cerebral angiography: prospective analysis of 2,899 procedures and review of the literature. Radiology 227:522-528

10. Aralasmak A, Akyuz M, Ozkaynak C, Sindel T, Tuncer R (2009) $\mathrm{CT}$ angiography and perfusion imaging in patients with subarachnoid hemorrhage: correlation of vasospasm to perfusion abnormality. Neuroradiology 51:85-93

11. Cho YD, Park JC, Kwon BJ, Hee HM (2010) Endovascular treatment of largely thrombosed saccular aneurysms: follow-up results in ten patients. Neuroradiology 52:751-758

12. Agid R, Willinsky RA, Lee SK, terBrugge KG, Farb RI (2008) Characterization of aneurysm remnants after endovascular treatment: contrast-enhanced MR angiography versus catheter digital subtraction angiography. AJNR Am J Neuroradiol 29:1570-1574

13. Uysal E, Oztora F, Ozel A, Erturk SM, Yildirim H, Basak M (2008) Detection and evaluation of intracranial aneurysms with 16-row multislice CT angiography: comparison with conventional angiography. Emerg Radiol 15:311-6

14. Krings T, Hans F (2004) New developments in MRA: timeresolved MRA. Neuroradiology 46(Suppl 2):214-22

15. Klingebiel R, Siebert E, Diekmann S, Wiener E, Masuhr F, Wagner M, Bauknecht HC, Dewey M, Bohner G (2009) 4-D Imaging in cerebrovascular disorders by using 320-slice CT: feasibility and preliminary clinical experience. Acad Radiol 16:123-129

16. Brouwer PA, Bosman T, van Walderveen MA, Krings T, Leroux AA, Willems PW (2010) Dynamic 320-section CT angiography in cranial arteriovenous shunting lesions. AJNR Am J Neuroradiol 31:767-770

17. Salomon EJ, Barfett J, Willems PW, Geibprasert S, Bacigaluppi S, Krings T (2009) Dynamic CT angiography and CT perfusion employing a 320-detector row CT: protocol and current clinical applications. Klin Neuroradiol 19:187-196

18. Saleh RS, Lohan DG, Villablanca JP, Duckwiler G, Kee ST, Finn JP (2008) Assessment of craniospinal arteriovenous malforma- 
tions at $3 \mathrm{~T}$ with highly temporally and highly spatially resolved contrast-enhanced MR angiography. AJNR Am J Neuroradiol 29:1024-1031

19. Willems PW, Brouwer PA, Barfett JJ, terBrugge KG, Krings T (2011) Detection and classification of cranial dural arteriovenous fistulas using 4D-CT angiography: initial experience. AJNR Am J Neuroradiol 32:49-53

20. Buis DR, Lagerwaard FJ, Barkhof F, Dirven CM, Lycklama GJ, Meijer OW, van den Berg R, Langendijk HA, Slotman BJ, Vandertop WP (2005) Stereotactic radiosurgery for brain AVMs: role of interobserver variation in target definition on digital subtraction angiography. Int J Radiat Oncol Biol Phys 62:246-252

21. Bambakidis NC, Sunshine JL, Faulhaber PF, Tarr RW, Selman WR, Ratcheson RA (2001) Functional evaluation of arteriovenous malformations. Neurosurg Focus 11:e2

22. Takeshita G, Toyama H, Nakane K, Nomura M, Osawa H, Ogura Y, Katada K, Takeuchi A, Koga S, Kato Y (1994) Evaluation of regional cerebral blood flow changes on perifocal brain tissue SPECT before and after removal of arteriovenous malformations. Nucl Med Commun 15:461-468 